\title{
Global burden of stroke and risk factors in 188 countries, during 1990-2013: a systematic analysis for the Global Burden of Disease Study 2013
}

\begin{abstract}
Valery L Feigin, Gregory A Roth, Mohsen Naghavi, Priya Parmar, Rita Krishnamurthi, Sumeet Chugh, George A Mensah, Bo Norrving, Ivy Shive, Marie Ng, Kara Estep, Kelly Cercy, Christopher J Murray, Mohammad H Forouzanfar, for the Global Burden of Diseases, Injuries, and Risk Factors Study 2013 and Stroke Experts Writing Group*
\end{abstract}

\begin{abstract}
Summary
Background The contribution of modifiable risk factors to the increasing global and regional burden of stroke is unclear, but knowledge about this contribution is crucial for informing stroke prevention strategies. We used data from the Global Burden of Disease Study 2013 (GBD 2013) to estimate the population-attributable fraction (PAF) of stroke-related disability-adjusted life-years (DALYs) associated with potentially modifiable environmental, occupational, behavioural, physiological, and metabolic risk factors in different age and sex groups worldwide and in high-income countries and low-income and middle-income countries, from 1990 to 2013.
\end{abstract}

Methods We used data on stroke-related DALYs, risk factors, and PAF from the GBD 2013 Study to estimate the burden of stroke by age and sex (with corresponding 95\% uncertainty intervals [UI]) in 188 countries, as measured with strokerelated DALYs in 1990 and 2013. We evaluated attributable DALYs for 17 risk factors (air pollution and environmental dietary, physical activity, tobacco smoke, and physiological) and six clusters of risk factors by use of three inputs: risk factor exposure, relative risks, and the theoretical minimum risk exposure level. For most risk factors, we synthesised data for exposure with a Bayesian meta-regression method (DisMod-MR) or spatial-temporal Gaussian process regression. We based relative risks on meta-regressions of published cohort and intervention studies. Attributable burden for clusters of risks and all risks combined took into account evidence on the mediation of some risks, such as high body-mass index (BMI), through other risks, such as high systolic blood pressure (SBP) and high total cholesterol.

Findings Globally, $\mathbf{9 0} \cdot 5 \%$ (95\% UI 88.5-92.2) of the stroke burden (as measured in DALYs) was attributable to the modifiable risk factors analysed, including $74 \cdot 2 \%$ (95\% UI $70 \cdot 7-76 \cdot 7)$ due to behavioural factors (smoking, poor diet, and low physical activity). Clusters of metabolic factors (high SBP, high BMI, high fasting plasma glucose, high total cholesterol, and low glomerular filtration rate; $72 \cdot 4 \%, 95 \%$ UI $70 \cdot 2-73 \cdot 5$ ) and environmental factors (air pollution and lead exposure; $33 \cdot 4 \%, 95 \%$ UI $32 \cdot 4-34 \cdot 3$ ) were the second and third largest contributors to DALYs. Globally, 29.2\% (95\% UI 28.2-29.6) of the burden of stroke was attributed to air pollution. Although globally there were no significant differences between sexes in the proportion of stroke burden due to behavioural, environmental, and metabolic risk clusters, in the low-income and middle-income countries, the PAF of behavioural risk clusters in males was greater than in females. The PAF of all risk factors increased from 1990 to 2013 (except for second-hand smoking and household air pollution from solid fuels) and varied significantly between countries.

Interpretation Our results suggest that more than $\mathbf{9 0 \%}$ of the stroke burden is attributable to modifiable risk factors, and achieving control of behavioural and metabolic risk factors could avert more than three-quarters of the global stroke burden. Air pollution has emerged as a significant contributor to global stroke burden, especially in lowincome and middle-income countries, and therefore reducing exposure to air pollution should be one of the main priorities to reduce stroke burden in these countries.

Funding Bill \& Melinda Gates Foundation, American Heart Association, US National Heart, Lung, and Blood Institute, Columbia University, Health Research Council of New Zealand, Brain Research New Zealand Centre of Research Excellence, and National Science Challenge, Ministry of Business, Innovation and Employment of New Zealand.

\section{Introduction}

A comparative assessment of the burden of stroke (in disability-adjusted life-years [DALYs]) attributable to potentially modifiable risk factors between regions and countries is crucial for education campaigns, evidencebased planning, priority setting, and resource allocation in stroke prevention. Understanding of the contribution of each risk factor to changes in stroke burden is important to establish country-specific and region-specific policies for both short-term and long-term population welfare. Documenting how much of the worldwide burden of stroke can be explained by lifestyle, environmental and occupational exposures, and metabolic risk factors allows quantification of the magnitude of stroke burden associated with various risks and prioritisation of preventive strategies at the global, regional, and national levels.
Lancet Neurol 2016; 15: 913-24 Published Online June 9, 2016 http://dx.doi.org/10.1016/ S1474-4422(16)30073-4 See Comment page 892 *Members listed at the end of the Article

National Institute for Stroke and Applied Neurosciences, Faculty of Health and Environmental Studies, Auckland University of Technology, Auckland, New Zealand (Prof V L Feigin MD, P Parmar PhD,

R Krishnamurthi PhD); Institute for Health Metrics and Evaluation, University of Washington, Seattle, WA, USA (GA Roth MD

Prof M Naghavi MD, SChugh MD, $M$ Ng PhD, K Estep BA K Cercy BA, Prof CJ L Murray MD Prof M H Forouzanfar PhD); Center for Translation Research and Implementation Science (CTRIS), National Heart, Lung, and Blood Institute, National Institutes of Health, Bethesda, MD, USA (G A Mensah MD); Department of Clinical Sciences, Neurology, Lund University , Lund, Sweden

(Prof B Norrving MD); Faculty of Health and Life Sciences, Northumbria University, Newcastle-upon-Tyne, UK (I Shive PhD); and Alzheimer Scotland Dementia Research Centre, University of Edinburgh, Edinburgh, UK (I Shive)

Correspondence to: Prof Valery L Feigin, National Institute for Stroke and Applied Neurosciences, School of Public Health and Psychosocial Studies, Faculty of Health and Environmental Studies, Auckland University of Technology, North Shore Campus, Auckland 0627, New Zealand valery.feigin@aut.ac.nz 
Research in context

\section{Evidence before this study}

Disability-adjusted life-years (DALYs) are the most comprehensive measure of population health, and combine the disability and mortality associated with a disease into one metric. We searched Scopus, MEDLINE, and PubMed for reports published in any language up to Dec 31, 2015, using the search terms "stroke" AND "DALY(s)" AND "population-attributable fraction" (PAF) AND "risk factors". A report from the INTERSTROKE study was based on findings from case-control studies in 22 countries and showed that more than $90 \%$ of strokes can be attributed to ten key stroke risk factors. The most recent Global Burden of Disease Study 2013 report provided a global, regional, and national comparative risk assessment of 79 behavioural, environmental and occupational, and metabolic risks factors or clusters of risk factors in 188 countries from 1990 to 2013 , but did not analyse in detail the stroke burden attributable to risk factors.

\section{Added value of this study}

To our knowledge this study is the first to quantify stroke burden in terms of DALYs and report changes in burden attributable to 17 potentially modifiable behavioural, environmental and occupational, and metabolic risks or clusters of risks at the global, regional, and country levels from 1990 to 2013 . The study showed that $90.5 \%$ (95\% UI 88.5-92.2) of the global burden of stroke was attributable to the modifiable risk factors analysed, including $74 \cdot 2 \%$ (95\% UI 70.7-76.7\%) to behavioural factors (ie, smoking, poor diet, and low physical activity). Clusters of metabolic factors (high systolic blood pressure, high body-mass index, high fasting plasma glucose, high total cholesterol, and low glomerular filtration rate) and environmental factors (air pollution and lead exposure) were the second and third largest contributors to DALYs. For the first time, air pollution emerged as one of the leading contributors to stroke burden worldwide, accounting for $29.2 \%$ of the stroke-related DALYs. Globally, the PAF of all risk factors to the burden of stroke increased with time (except for second-hand smoking and household air pollution from solid fuels) and varied significantly between countries and regions.

\section{Implications of all the available evidence}

These findings are important for education campaigns, evidence-based planning, priority setting (including for stroke research), and resource allocation in stroke prevention. Understanding the contribution of each risk factor and risk cluster to the changes in stroke burden is important to establish country-specific and region-specific policies on stroke prevention strategies. Although the proportion of the stroke burden of risk clusters provides a broad view of investment priorities, stroke burden due to individual risks in different age groups of the population can inform the potential elements of a more specific intervention. For example, reducing exposure to air pollution should be one of the main priorities to reduce stroke burden in low-income and middle-income countries, whereas reduction of behavioural risks should be one of the main priorities in high-income countries. For future research, the next step is to close the knowledge gap on stroke burden. This would include identification of previous health conditions grouped by age, sex, and stroke subtype, in addition to the regular monitoring of stroke incidence, mortality, prevalence, associated disability, and modifiable risk factors.
Previously, we reported on 79 behavioural, environmental and occupational, and metabolic risks or clusters of risks for diseases, including all cardiovascular disease, in 188 countries from 1990 to 2013. ${ }^{1}$ In this Article, we report estimates from the Global Burden of Disease Study 2013 (GBD 2013) for population-attributable fraction (PAF) of stroke-related DALYs associated with potentially modifiable environmental and occupational, behavioural, and physiological, and metabolic risk factors by age groups globally, in high-income countries, and in low-income and middle-income countries as defined by the World Bank, from 1990 to 2013. We also report PAF of stroke-related DALYs associated with behavioural, environmental, and metabolic risk clusters by sex, globally, and regions in 2013.

\section{Methods \\ Overview}

Details about the basic approach for the GBD 2013 estimates of the PAF and burden of stroke have been reported previously. ${ }^{1-5}$ Briefly, we used published and unpublished data on the distribution of risks to estimate the fraction of the population in each country, age group, and year exposed to different levels of risk factors, when evidence of a causal and biologically plausible association between exposure to the risk factor and the incidence of stroke was convincing. ${ }^{1}$ GBD 2013 provides the rates of stroke-related mortality, years of life lost, years lost because of disabilities, and DALYs. ${ }^{3,6,7}$ Citations for all sources used to estimate risk factor exposure have been reported in GBD $2013^{1}$ and Roth and colleagues ${ }^{4}$ provide citations for all sources used to estimate stroke-related DALYs by country. We defined convincing evidence as evidence from epidemiological studies (prospective observational studies and randomised controlled trials of sufficient size, duration, and quality) showing consistent associations between exposure and disease, with little or no evidence to the contrary. For some risks, such as diet and alcohol consumption, we also used administrative record systems. Of 79 behavioural, environmental and occupational, and metabolic risks or clusters of risks analysed in GBD 2013, ${ }^{1}$ we selected only those for which stroke was included as a relevant outcome, and for which sufficient data were available to estimate risk factor 


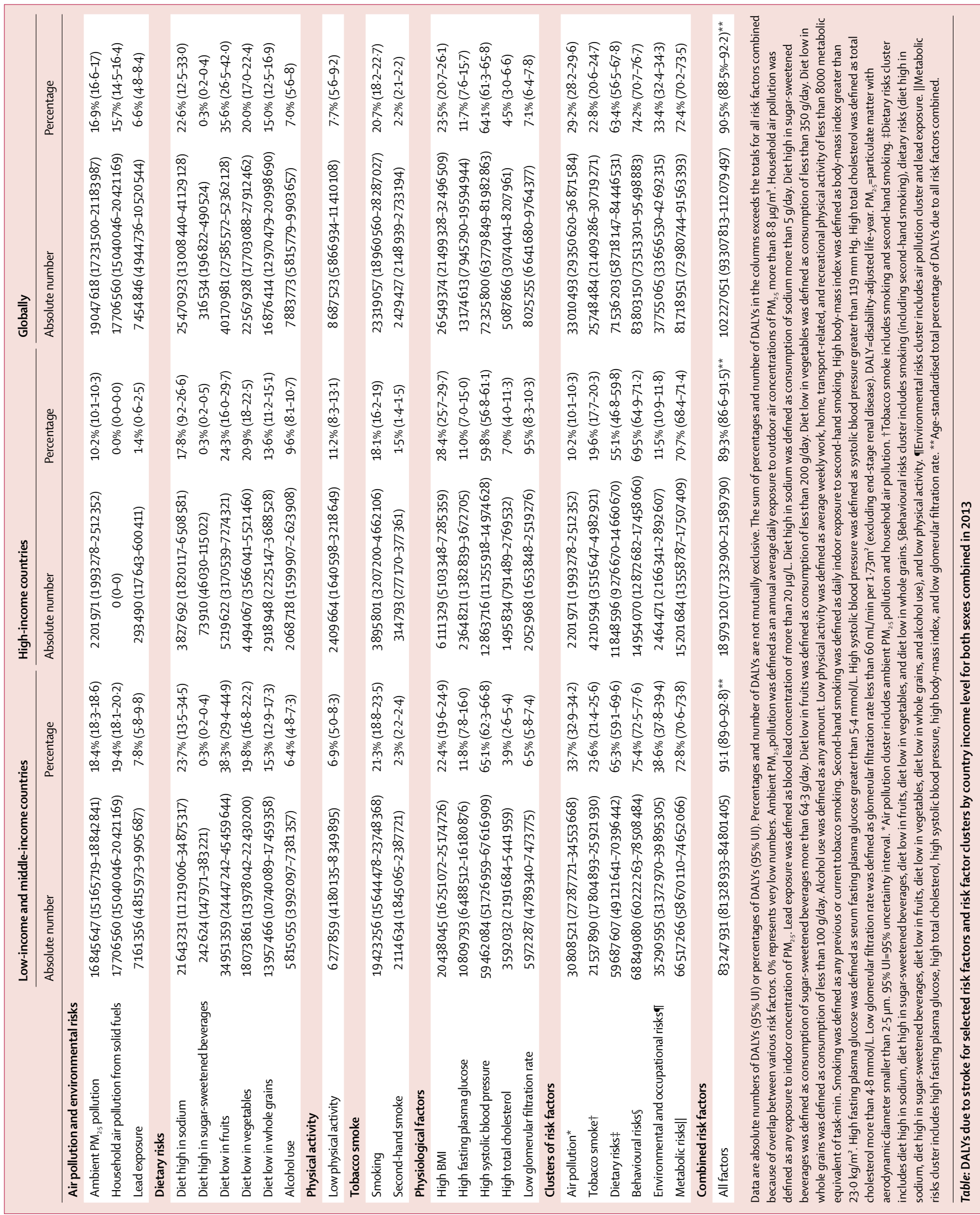


exposure. We defined sufficient data as estimates for which the effect sizes increase per unit of exposure, and for which there was evidence that these effects could be applied to a general population. We assessed risk-outcome pairs (17 risks and stroke-related DALYs) that met explicit evidence criteria for 188 countries.

\section{Stroke burden and risk factors}

To estimate stroke burden associated with various risks, we included any exposure to risks that exceeded a theoretical minimum risk exposure level (TMREL). TMREL was defined as the level of exposure at which risk from all causes of DALYs combined is at a minimum, and was based on a consultation with risk factor epidemiologists. As explained in detail in GBD 2013, ${ }^{1}$ the TMREL should minimise individual (and population level) risk and should, in theory, be possible to achieve, but not necessarily affordable or feasible. For causes such as systolic blood pressure (SBP) of less than $120 \mathrm{~mm} \mathrm{Hg}$, the evidence for the selection of the TMREL is convincing. ${ }^{8}$ For other causes, such as sodium consumption or body-mass index (BMI), the evidence for the selection of the TMREL is not so certain and, for these causes, we used a uniform distribution of different TMRELs in the uncertainty estimation sampling, as explained in detail previously. Based on the selected TMRELs, we established 17 risks: ambient particulate matter pollution, household air pollution from solid fuels, lead exposure, diet high in sodium, diet high in sugar-sweetened beverages, diet low in fruits, diet low in vegetables, diet low in whole grains, alcohol consumption (any dosage), low physical activity, smoking, second-hand smoking, high BMI, high fasting plasma glucose, high SBP, high total cholesterol, and low glomerular filtration rate (GFR). The criteria used to define these risks are reported in the table. We also assessed clusters of risk factors: behavioural (ie, smoking [including second-hand smoking], dietary [diet high in sodium, diet high in sugar-sweetened beverages, diet low in fruits, diet low in vegetables, diet low in whole grains, and alcohol use], low physical activity), environmental and occupational (ie, ambient particulate matter pollution, household air pollution, and lead exposure), and metabolic (high BMI, high fasting plasma glucose, high SBP, high total cholesterol, high GFR). Tobacco smoke (smoking and second-hand smoking) was considered as a separate cluster but was also included in the behavioural cluster. The PAFs of risk factor clusters took into account interactions between risk factors included in the cluster, as explained elsewhere. ${ }^{2}$

\section{Statistical analysis}

See Online for appendix

For most risks, we synthesised data for exposure with a Bayesian meta-regression method, DisMod-MR $2.0^{\circ}$ or spatial-temporal Gaussian process regression..$^{10}$ Relative risks were based on meta-regressions of published cohort and intervention studies. Attributable burden for clusters of risks and all risks combined took into account evidence on the mediation of some risks, such as high BMI, through other risks, such as high SBP and high total cholesterol. Justifications for thresholds of the risks have been discussed in the GBD 2013 report. The relative risks were summarised by age and sex for each risk factor and outcome pair. ${ }^{1}$ As explained previously, ${ }^{1}$ we used relative risks from studies controlled for confounding but not for factors along the causal pathway between exposure and outcome. We used estimates of the size of the association between exposure to a risk factor and stroke (rate ratios) ${ }^{11}$ and estimates of stroke-related DALYs from GBD 2013 ${ }^{1,3}$ to calculate the burden (as measured in DALYs) attributable to each risk factor exposure in view of the TMREL (PAF analysis). This approach brings together data for excess stroke mortality and disability associated with risk factors, data for exposure to risks, and evidence-based assumptions on the desired counterfactual distribution of risk exposure to estimate how much of the stroke burden in a particular year can be attributed to risk exposure in that year and in all previous years. PAF is the estimated proportion of disease burden in a population that would be avoided if exposure to a risk factor were reduced to an alternative theoretical exposure scenario (eg, no tobacco use). The attributable stroke burden was calculated using the equation:

$A B_{\text {jasct }}=\sum^{W} D A L Y_{\text {oast }} P A F_{\text {joasct }}$

Where $A B_{\text {jast }}$ is the attributable burden for risk factor $j$ in age group $a$, sex $s$, country $c$, and year $t$. DALY $Y_{\text {oasc }}$ is DALYs for cause $o$ (of $w$ relevant outcomes for risk factors $j$ ) in age group $a$, sex $s$, country $c$, and year $t$. $P A F_{\text {joast }}$ is the PAF for cause $o$ due to risk factor $j$ in age group $a$, sex $s$, country $c$, and year $t$. Risk factors were assessed individually and then aggregated in multiple hierarchical levels including environmental, behavioural, physiological, and metabolic categories of individual risk factors. We assumed the risks to be independent, but mediators between risks were included if data were available. ${ }^{1}$ PAFs of risk factor clusters took into account interactions between risk factors included in the cluster, as explained elsewhere. ${ }^{2}$ Reported estimates for risk factors and risk factor clusters are not mutually exclusive or adjusted for other risk factors or risk factor clusters analysed. We stratified countries into two groups (highincome countries and low-income and middle-income countries based on the gross domestic product). ${ }^{12}$ Countries included in various GBD regions are listed in the appendix (pp 1-2).

To estimate the proportion (\%) of DALYs associated with the analysed individual risk factors and risk factor clusters, we used stroke-related DALYs from GBD 2013 $3^{1,3,7}$ at global, 
regional, and country-specific levels. We used the same methods as used for GBD $2013^{1}$ to calculate uncertainty intervals (UI) based on a Bayesian approach for all PAFs. UIs should be interpreted as given current data and prior assumptions (priors), and the statistics might vary by this interval. Contrary to the frequentist approach commonly used to estimate $95 \%$ CIs, our approach does not assume a single true value for $95 \% \mathrm{CI}$, but instead has a posterior distribution. For practical purposes, the implications of both UIs and CIs are similar. The inclusion criteria for each risk-outcome (stroke) pair, data sources, and methods of a systematic search to identify relevant scientific literature to estimate risk factor exposure distributions in 1990 and 2013 have been described in detail elsewhere. ${ }^{1,4}$

\section{Role of the funding source}

The funder of the study had no role in study design, data collection, data analysis, data interpretation, or writing of the report. The corresponding author had full access to all the data in the study and had final responsibility for the decision to submit for publication.

\section{Results}

Globally, $90 \cdot 5 \%$ of the burden of stroke (as measured in DALYs) was attributable to the modifiable risk factors analysed (table). Although, in most countries worldwide, stroke burden from all these risk factors combined varied between $80 \%$ and $97 \%$; in most African countries, it varied between $72 \%$ and $79 \%$ (figure 1 ). Of the total

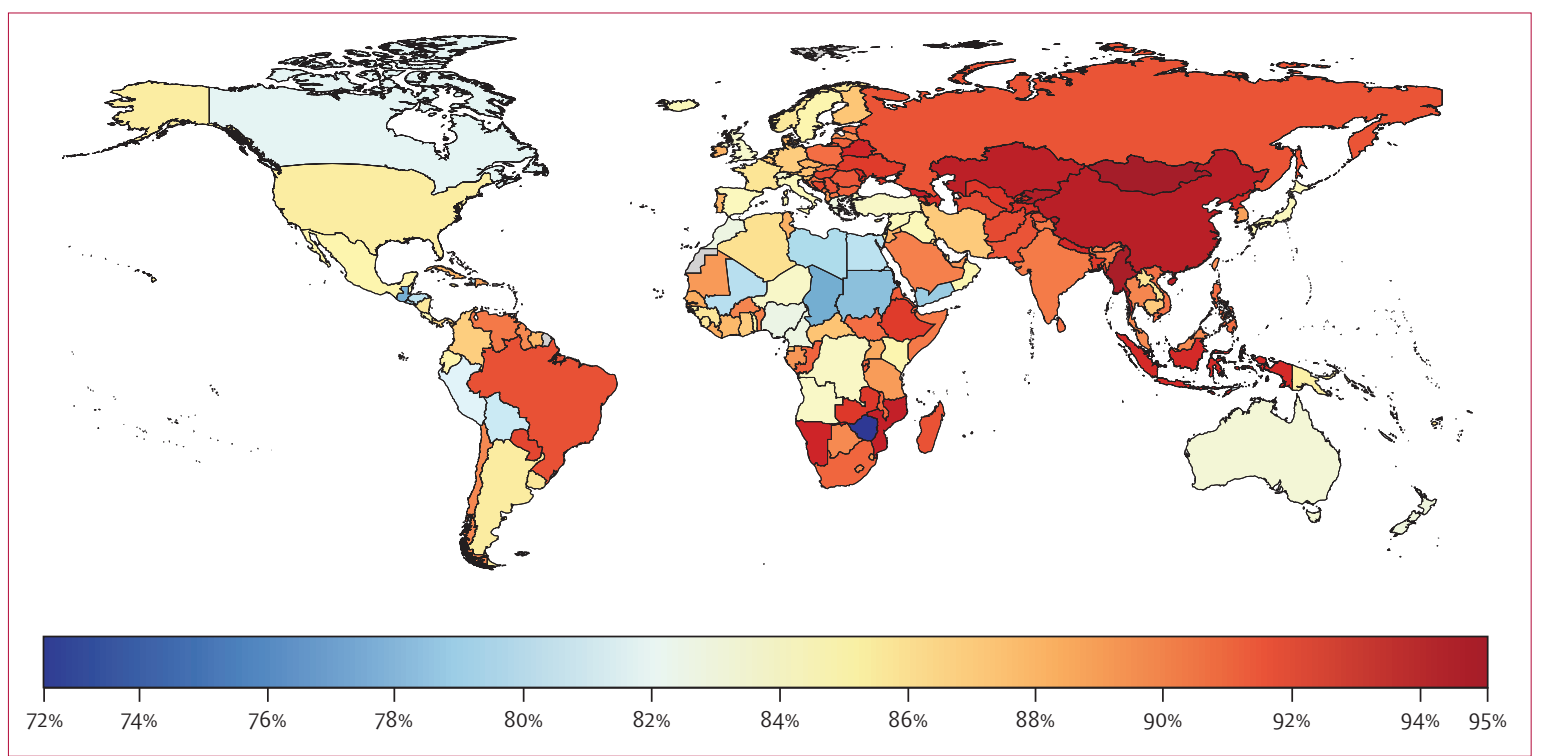

Figure 1: Stroke-related DALYs attributable to all modifiable risk factors combined for both sexes in 2013

DALY=disability-adjusted life-year.

\begin{tabular}{|c|c|c|c|c|c|c|c|c|c|c|c|c|c|c|c|c|c|c|c|c|c|c|c|}
\hline $\begin{array}{l}\text { Rank } \\
\square \text { 1-5 } \\
\square \text { 6-10 }\end{array}$ & $\begin{array}{l}\square \text { 11-15 } \\
\square>15\end{array}$ & $\begin{array}{l}\bar{\pi} \\
\frac{0}{0} \\
\frac{0}{v}\end{array}$ & 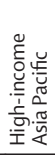 & 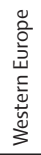 & 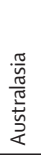 & 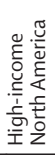 & 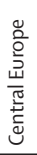 & 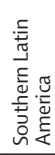 & 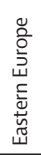 & 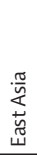 & 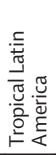 & 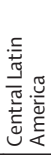 & 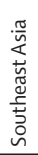 & 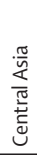 & 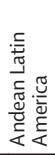 & 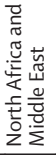 & 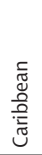 & $\begin{array}{l}\frac{\pi}{2} \\
\frac{1}{4} \\
\frac{1}{5} \\
0 \\
0\end{array}$ & 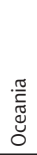 & 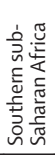 & 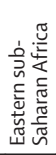 & 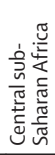 & 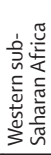 \\
\hline \multicolumn{2}{|c|}{ High systolic blood pressure } & 1 & 1 & 1 & 1 & 1 & 1 & 1 & 1 & 1 & 1 & 1 & 1 & 1 & 1 & 1 & 1 & 1 & 1 & 1 & 1 & 1 & 1 \\
\hline \multicolumn{2}{|c|}{ Diet low in fruits } & 2 & 2 & 4 & 4 & 3 & 4 & 4 & 3 & 2 & 3 & 4 & 2 & 2 & 3 & 3 & 3 & 2 & 3 & 2 & 2 & 2 & 2 \\
\hline \multicolumn{2}{|c|}{ High body-mass index } & 3 & 6 & 2 & 2 & 2 & 2 & 2 & 2 & 6 & 2 & 2 & 6 & 3 & 2 & 2 & 2 & 8 & 2 & 3 & 5 & 5 & 4 \\
\hline \multicolumn{2}{|c|}{ Diet high in sodium } & 4 & 3 & 6 & 10 & 6 & 3 & 8 & 6 & 3 & 4 & 8 & 8 & 4 & 7 & 4 & 12 & 5 & 13 & 7 & 9 & 11 & 8 \\
\hline \multicolumn{2}{|l|}{ Smoking } & 5 & 4 & 5 & 5 & 5 & 6 & 5 & 5 & 4 & 6 & 6 & 4 & 6 & 6 & 8 & 5 & 7 & 5 & 5 & 8 & 10 & 12 \\
\hline \multicolumn{2}{|c|}{ Diet low in vegetables } & 6 & 5 & 3 & 3 & 4 & 5 & 3 & 4 & 11 & 5 & 3 & 3 & 5 & 4 & 5 & 4 & 4 & 4 & 4 & 4 & 4 & 5 \\
\hline \multicolumn{2}{|c|}{ Ambient particulate matter $(2.5 \mu \mathrm{m})$ pollution } & 7 & 8 & 11 & 14 & 12 & 8 & 12 & 10 & 5 & 9 & 9 & 9 & 8 & 9 & 6 & 11 & 6 & 15 & 11 & 12 & 6 & 6 \\
\hline \multicolumn{2}{|c|}{ Household air pollution from solid fuels } & 8 & .. & .. & .. & .. & ... & 15 & .. & 7 & 14 & 12 & 5 & 12 & 11 & 14 & 7 & 3 & 6 & 8 & 3 & 3 & 3 \\
\hline \multicolumn{2}{|c|}{ Diet low in whole grains } & 9 & 7 & 9 & 8 & 9 & 7 & 6 & 7 & 8 & 7 & 5 & 7 & 7 & 5 & 7 & 6 & 10 & 7 & 10 & 10 & 7 & 9 \\
\hline \multicolumn{2}{|c|}{ High fasting plasma glucose } & 10 & 10 & 10 & 9 & 7 & 11 & 7 & 9 & 9 & 8 & 7 & 10 & 9 & 10 & 9 & 10 & 9 & 8 & 9 & 11 & 8 & 11 \\
\hline \multicolumn{2}{|c|}{$\underline{\text { Low physical activity }}$} & 11 & 9 & 8 & 6 & 8 & 10 & 11 & 11 & 13 & 11 & 13 & 11 & 13 & 12 & 10 & 8 & 12 & 9 & 13 & 14 & 12 & 10 \\
\hline \multicolumn{2}{|c|}{ Low glomerular filtration rate } & 12 & 12 & 7 & 7 & 13 & 9 & 10 & 12 & 14 & 10 & 10 & 14 & 10 & 8 & 11 & 9 & 11 & 12 & 6 & 7 & 9 & 7 \\
\hline \multicolumn{2}{|l|}{ Alcohol use } & 13 & 11 & 12 & 11 & 10 & 12 & 9 & 8 & 12 & 12 & 11 & 15 & 11 & 14 & 16 & 15 & 15 & 11 & 12 & 13 & 14 & 14 \\
\hline \multicolumn{2}{|c|}{ Lead exposure } & 14 & 14 & 14 & 13 & 15 & 14 & 16 & 15 & 10 & 15 & 16 & 12 & 14 & 15 & 13 & 13 & 13 & 14 & 14 & 6 & 13 & 13 \\
\hline \multicolumn{2}{|c|}{ High total cholesterol } & 15 & 13 & 13 & 12 & 11 & 13 & 13 & 13 & 16 & 13 & 14 & 13 & 15 & 13 & 12 & 14 & 14 & 10 & 15 & 15 & 15 & 15 \\
\hline \multicolumn{2}{|c|}{ Second-hand smoke } & 16 & 15 & 15 & 15 & 16 & 15 & 14 & 14 & 15 & 16 & 17 & 16 & 16 & 17 & 15 & 17 & 16 & 16 & 16 & 16 & 16 & 16 \\
\hline \multicolumn{2}{|c|}{ Diet high in sugar-sweetened beverages } & 17 & 16 & 16 & 16 & 14 & 16 & 17 & 16 & 17 & 17 & 15 & 17 & 17 & 16 & 17 & 16 & 17 & 17 & 17 & 17 & 17 & 17 \\
\hline
\end{tabular}

Figure 2: Top risk factors ranked by number of DALYs attributable to stroke for both sexes combined in 21 regions in 2013

For the list of countries included within these regions, please see the appendix (pp 1-2). DALY=disability-adjusted life-year. 
stroke-related DALYs, 74.2\% (83.8 million DALYs) were due to behavioural factors (ie, smoking, poor diet, and low physical activity) and $72.4 \%$ (81.7 million DALYs) were attributable to metabolic risks (ie, high fasting plasma glucose, high total cholesterol, high SBP, high BMI, and low GFR; table). The five leading risk factors for DALYs were high SBP (72.3 million DALYs [64.1\%]), diet low in fruits $(40 \cdot 2$ million DALYs [35.6\%]), high BMI (26.5 million DALYs [23.5\%]), diet high in sodium (25. 5 million DALYs [22.6\%]), and smoking (23.3 million DALYs [20.7\%]; figure 2, 3, table). Clusters of metabolic and behavioural risk factors were the leading causes of stroke-related DALYs in low-income and middle-income countries (15.2 million DALYs [70.7\%] and $15 \cdot 0$ million DALYs [69.5\%]; table).

We noticed geographical variations in the highest and lowest PAFs of risk factors associated with stroke-related DALYs (appendix p 3). For example, stroke burden associated with household air pollution from solid fuels was highest in central, eastern, and western subSaharan Africa and south Asia, and lowest in highincome North America, central, eastern, and western Europe, Australasia, and high-income Asia Pacific. Almost half the stroke-related DALYs due to the risk factors and risk factor clusters occurred in people aged 50-69 years (appendix p 7). Although most risk factors accounted for more DALYs in people aged 15-49 years than in people aged 70 years or older, the PAFs of low physical activity, low GFR, and lead exposure were greater in the older age group (appendix pp 4-9). Globally, household air pollution from solid fuels, diet low in vegetables, high BMI, air pollution, and environmental risk clusters accounted for significantly more DALYs in people aged 15-49 years than in the other age groups, but high SBP and metabolic risk clusters were most prominent in people aged 50-69 years globally and in low-income and middleincome countries (appendix pp 4-6).

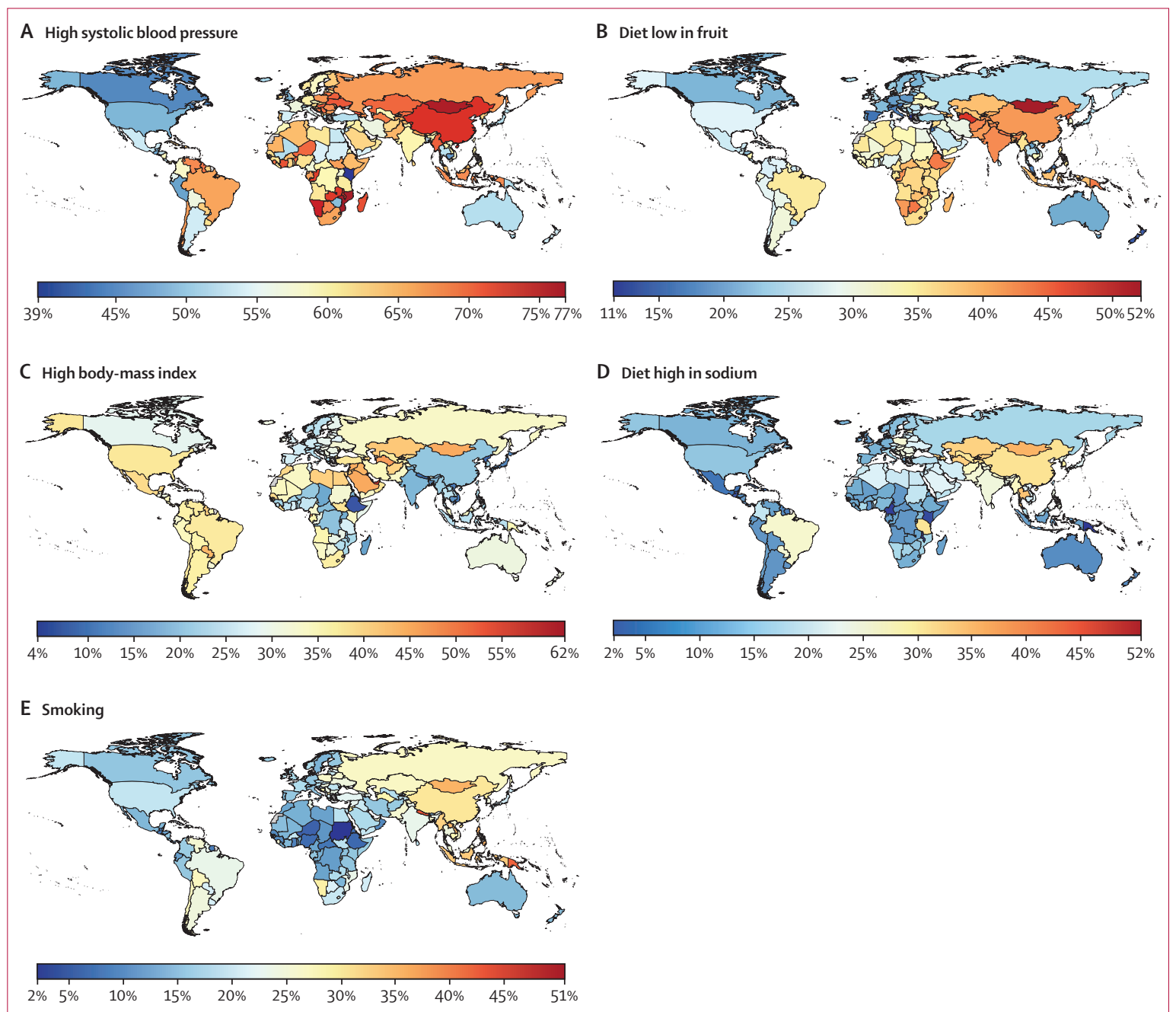

Figure 3: Global distribution of leading risk factors for stroke-related DALYs in 2013

Maps show the five leading risk factors for stroke-related DALYs (as established by PAF) for both sexes combined in 2013: high systolic blood pressure (A), diet low in fruit (B), high body-mass index (C), diet high in sodium (D), and smoking (E). DALY=disability-adjusted life-year. PAF=population-attributable fraction. 
Globally, during 1990-2013, we noticed increases in the stroke-related burden of almost all individual risk factors (appendix p 10). The only risk factors with a decrease in stroke-related DALYs were second-hand smoke $(-31.0 \%$, $95 \%$ UI $-31 \cdot 3$ to $-30 \cdot 7)$ and household air pollution from solid fuels $(-1.9 \%,-4 \cdot 2$ to $-0.4 \%)$. Globally, there was a significant increase in the stroke-related DALYs associated with high BMI (>46\% increase), low physical activity $(>39 \%$ increase), high fasting plasma glucose ( $44 \%$ increase), high SBP (>39\% increase), diet high in sugar-sweetened beverages ( $>63 \%$ increase), high total cholesterol ( $24 \%$ increase), ambient particulate matter pollution of aerodynamic diameter less than $2.5 \mu \mathrm{m}$ $\left(\mathrm{PM}_{2.5}\right)$ (>33\% increase), alcohol use (>32\% increase), low GFR ( $25 \%$ increase), diet high in sodium (>34\% increase), diet low in vegetables (23\% increase), and smoking ( $>10 \%$ increase). The only risk factors that were significantly reduced globally were household air pollution from solid fuels ( $2 \%$ reduction) and secondhand smoke (31\% reduction). Changes in other risk factors were not significant on the global scale. In highincome countries, there were significant reductions in stroke-related DALYs due to ambient air pollution, household air pollution from solid fuels, lead exposure, diet high in sodium, diet low in fruits, vegetables, and whole grains, smoking, second-hand smoking, alcohol use, low physical activity, high BMI, high fasting plasma glucose, high SBP, high total cholesterol, and low GFR. However, there was a large (almost 84\%) and significant increase in diet high in sugar-sweetened beverages. In low-income and middle-income countries, there were significant increases in stroke-related DALYs due to ambient air pollution, all dietary risks and alcohol use, low physical activity, smoking, high BMI, high fasting plasma glucose, high SBP, high total cholesterol, and low GFR.

Globally, in 2013, the proportions of stroke burden due to behavioural, environmental, and metabolic risk clusters were non-significantly higher in males than in females (figure 4; appendix pp 11-12). However, in 2013, differences between the sexes were significant for the PAF of stroke-related age-standardised DALYs attributable to the behavioural risk cluster in low-income and middle-income countries (1376 per 100000 personyears and $79.2 \%$ in males vs 972 per 100000 person-years and $70.3 \%$ in females), but difference in high-income countries was not significant (1263 per 100000 personyears and $73.2 \%$ in males vs 1014 per 100000 personyears and $68 \cdot 1 \%$ in females; appendix pp 11-12).

In high-income countries in 2013, the five leading risk factors for DALYs were high SBP, high BMI, diet low in fruits, diet low in vegetables, and smoking (table). Also, in these countries, metabolic and behavioural risk factor clusters were the first and second leading causes of stroke-related DALYs, accounting for 15.2 million DALYs $(70 \cdot 7 \%)$ and $15 \cdot 0$ million DALYs (69.5\%) (table). Globally, household air pollution from solid fuels, diet low in vegetables, high BMI, and air pollution and environmental risk clusters accounted for significantly more DALYs in people aged 15-49 years than in other age groups, and high SBP and the metabolic risk cluster were most prominent in people aged 50-69 years in high-income countries (appendix pp 4-5).

The five individual risk factors that contributed most to the stroke-related DALYs in low-income and middleincome countries were high SBP, diet low in fruits, diet high in sodium, high BMI, and smoking (table). The clusters of risk factors that contributed most to DALYs in low-income and middle-income countries were behavioural (68.8 million DALYs [75.4\%]; behavioural risks included dietary risks), metabolic $(66.5$ million DALYs [72.8\%]), and environmental risks (35.3 million DALYs [38.6\%]), of which ambient $\mathrm{PM}_{2.5}$ pollution accounted for $16 \cdot 9$ million DALYs $(18 \cdot 4 \%)$ and household air pollution from solid fuels for 17.7 million DALYs (19.4\%) (table).

Generally, most risk factors (except low physical activity, high total cholesterol, and low GFR) had the greatest effect on DALYs in low-income and middle-income countries (appendix pp 25-36), and large variations existed between countries in PAFs of the risk factors (figures 2, 3; appendix pp 13-24).
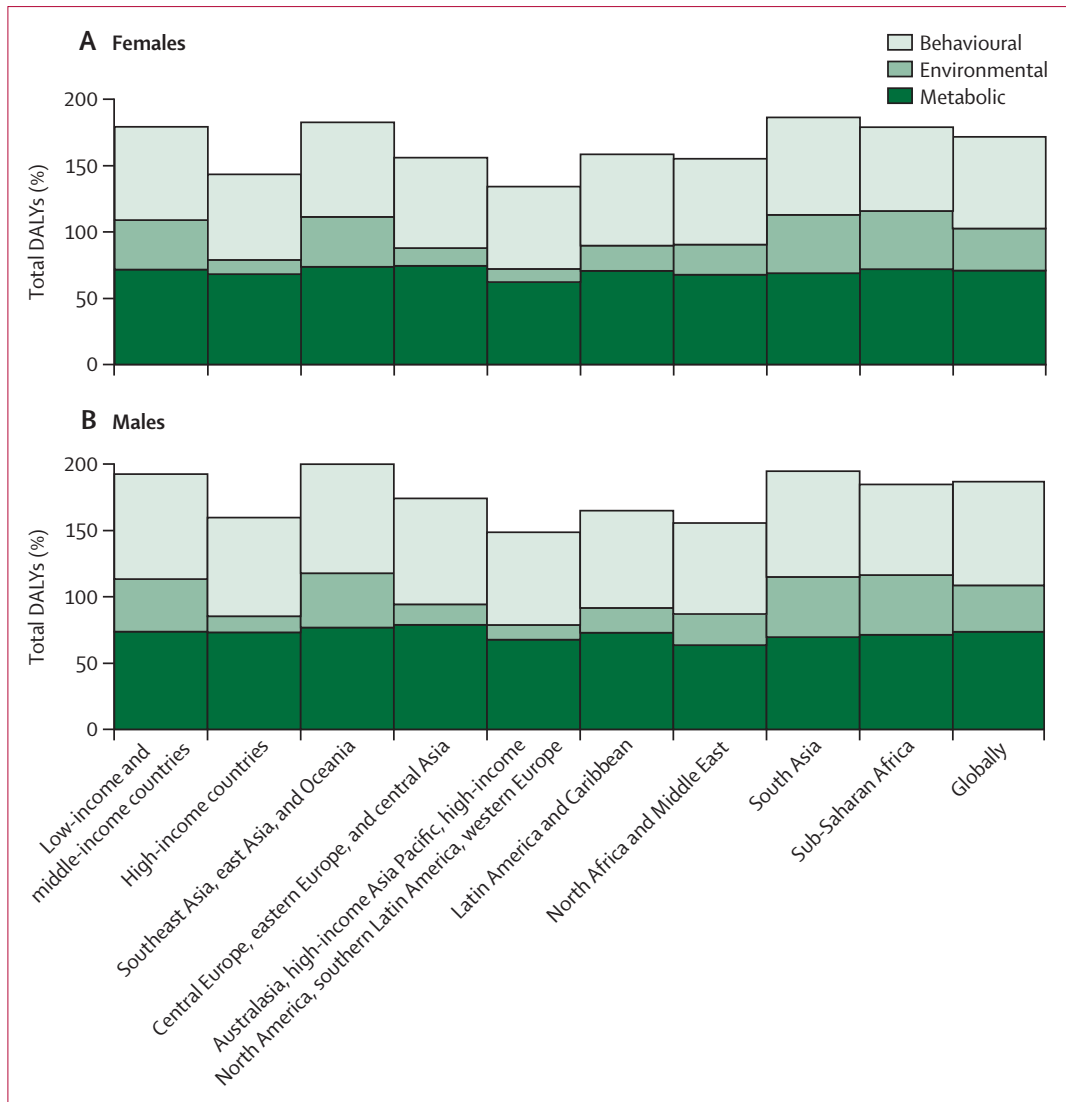

Figure 4: Stroke-related, age-standardised DALYs attributable to behavioural, environmental, and metabolic risk clusters by sex in 2013

DALY=disability-adjusted life-year. 


\section{Discussion}

Our results show the global, regional, and countryspecific burden of stroke (as measured with DALYs) associated with potentially modifiable risk factors and risk factor clusters from 1990 to 2013. For the first time, air pollution has emerged as one of the leading contributors to stroke burden worldwide, accounting for almost a third of stroke-related DALYs. Our findings suggest that more than $90 \%$ of the global burden of stroke in 2013 was attributable to the combined effect of all modifiable risk factors analysed, and that most of the burden of stroke is attributable to behavioural factors and metabolic factors. However, the PAF of stroke risk factors and risk factor clusters differed between countries and regions. For example, the extent of stroke burden attributable to air pollution and environmental risks, tobacco smoke, dietary risks, and high SBP in lowincome and middle-income countries was significantly greater (except for dietary risks) than in high-income countries, whereas the contribution of low physical activity and low GFR was significantly greater in highincome countries. Because many of the behavioural risk factors we analysed mediate their effects through metabolic risk factors, ${ }^{13}$ efforts to reduce exposure to behavioural risk factors will enhance the control of metabolic risk factors. Other studies ${ }^{14-16}$ have also reported an increasing role of some environmental and behavioural risk factors for stroke and geographical variations in mediating the effects of cardiovascular risk factors. Our results showing the key contributions of high SBP, diet low in fruits, high BMI, and smoking to stroke burden are in line with those of other studies. ${ }^{17-20}$ Furthermore, for the first time, our analyses showed diverging trends in the proportions of DALYs attributable to most modifiable risk factors from 1990 to 2013, with significant increases in low-income and middle-income countries and significant decreases in high-income countries. If these trends continue, the large disparities that exist in stroke burden between high-income and low-income and middle-income countries ${ }^{3}$ will be further increased. Our finding that, globally and in low-income and middle-income countries, diet high in sodium is one of the five leading risk factors for DALYs, with a contribution similar to that of tobacco smoking, represents a new way of looking at the ranking of risk factors in global stroke burden and can be explained by its causal role in elevated blood pressure. ${ }^{21}$ Our findings of the large effect of poor diet (especially diet low in fruits) on stroke burden are consistent with previous studies. ${ }^{22}$ Although a diet low in vegetables was ranked in the five most common risk factors for DALYs in every region except east Asia, it ranked sixth globally, showing that east Asia (especially China) has a large effect on the overall ranking of risk factors (figure 2). Changes in the PAF of smoking (decreases in tobacco smoking and second-hand smoking in high-income countries, decreases in second-hand smoking in low-income and middle-income countries, and increases in tobacco smoking in low-income and middle-income countries) during 1990-2013 could reflect corresponding changes in smoking prevalence and tobacco banning policies in high-income and low-income and middle-income countries. ${ }^{23-26}$ The paradox in low-income and middleincome countries of decreased second-hand smoking and increased tobacco smoking warrants further investigation. Although the contribution of high total cholesterol to DALYs was low $(4 \cdot 5 \%$ globally), it was consistent with the range of effects $(0-15 \%)$ in other studies. ${ }^{27}$

Our data show that, globally, differences between sexes in the proportion of stroke burden due to behavioural environmental, and metabolic risks were not significant. In low-income and middle-income countries (especially in east Asia) the PAF of the behavioural risk cluster was greater in males than in females. These findings suggest that although preventive interventions to reduce exposure to behavioural, environmental, and metabolic risks will have about the same effect on reducing stroke burden in both males and females in low-income and middleincome countries (especially in east Asia), preventive interventions to reduce exposure to behavioural risks are likely to produce greater reductions in stroke burden in males than in females. Sex differences in stroke burden were also shown in another GBD 2013. ${ }^{28}$

Our results are consistent with those of the INTERSTROKE study, ${ }^{18}$ in which roughly $86 \%$ of strokes could be attributed to nine potentially modifiable metabolic and behavioural risk factors, emphasising their importance for defining global and regional priorities in reducing stroke burden. Although the noticeable global epidemiological transition happening in low-income and middle-income countries ${ }^{1}$ (especially increasing exposure to western lifestyle) might largely be accountable for the greater contribution of behavioural risk factors to stroke burden in these countries, a striking finding of our study was an unexpectedly high proportion of stroke burden attributable to air pollution (29.2\% globally), especially in low-income and middle-income countries $(33.7 \%$ vs $10.2 \%$ in high-income countries). In 2013, air pollution contributed to about 33 million stroke-related DALYs, including almost 31 million DALYs in low-income and middle-income countries (table). For example, in south Asia and eastern and central sub-Saharan Africa, based on PAFs, air pollution was the third highest contributor to stroke-related DALYs, accounting for almost $40 \%$ of the DALYs (data not shown). In China and India, almost 22\% of stroke-related DALYs in 2013 were attributed to ambient $\mathrm{PM}_{2.5}$ air pollution. Although household air pollution from solid fuels did not contribute to stroke burden in high-income countries, almost a fifth of stroke burden in low-income and middle-income countries was attributed to household air pollution in 2013 (especially in Asia and sub-Saharan Africa; appendix p 27). These findings are in line with reports ${ }^{29,30}$ concluding that ambient and 
household air pollution is now the world's largest combined environmental health risk, although these reports were based on observational studies. Possible mechanisms of the association between stroke and air pollution include adverse effects of air pollution on the vascular endothelium, increases in blood pressure, and increased risk of thrombosis. ${ }^{29}$ Additionally, evidence suggests that not all $\mathrm{PM}_{2.5}$ pollution has the same cardiovascular implications, with fossil fuel emissions regarded as more damaging (especially coal burning) than other types. ${ }^{31}$ Consequently, we might be underestimating the effect of $\mathrm{PM}_{2.5}$ pollution on stroke in countries with greater contributions of such fossil fuel burning to the total $\mathrm{PM}_{2.5}{ }^{31}$ The contribution of lead exposure to stroke burden might be due to an interaction with hypertension ${ }^{32}$ and other cardiovascular risk factors. ${ }^{32}$

The proportion of DALYs attributable to the analysed risk factors and risk factor clusters varied substantially by age group, country economic status, and geographical region. The lower percentage of DALYs attributable to all risk factors combined in African countries compared with other countries might be related to infectious and rheumatic diseases having a greater role as causes of stroke in Africa than in other countries (these risk factors were not analysed in the present manuscript). ${ }^{33}$ For example, in sub-Saharan Africa low GFR might be a consequence of fairly high prevalence of HIV infection. ${ }^{34}$ Globally and in high-income countries, the PAF of all risk factors combined to DALYs was largest in people aged 50-69 years, followed by people aged $15-49$ years. In lowincome and middle-income countries, the proportion of PAF of all risk factors combined was also largest for people aged 50-69 years, but was almost the same in people aged $15-49$ years and older than 70 years. The high number of DALYs attributable to modifiable risk factors in people aged 15-49 years and 50-69 years in high-income countries shows an opportunity to halt the burden of stroke in high-income countries. The risk factor that was most increased from 1990 to 2013 was diet high in sugarsweetened beverages $(63.1 \%$ increase in stroke-related DALYs), with the greatest increase noted in high-income countries. Although the contribution of this risk factor to stroke burden is currently relatively low (about $0.3 \%$ ), given its association with overweight and type 2 diabetes mellitus, continuing exposure to diet high in sugarsweetened beverages could, in a couple of decades, result in a huge increase in stroke burden associated with this risk factor. The differences in rankings between lowincome and middle-income and high-income countries by age could represent changes in behaviours in more recent birth cohorts (eg, changes in the proportions of diet low in vegetables in each age group) in these countries and might help in prediction of the future burden. For example, in low-income and middle-income countries, high BMI was ranked ninth in people aged 70 years or older, but was third and fourth in the younger age groups (appendix $\mathrm{p}$ 9). The large percentage increase in DALYs due to some behavioural, dietary, and metabolic risk factors could be a reflection of the negative convergence of these risk factors on cardiovascular disease that has been noted in some countries. ${ }^{35}$

Although globally the ten most important risk factors were high SBP, diet low in fruits, high BMI, diet high in sodium, smoking, diet low in vegetables, ambient $\mathrm{PM}_{2.5}$ pollution, household air pollution from solid fuels, diet low in whole grains, and high fasting plasma glucose, there was much heterogeneity in the PAF of these risk factors for stroke-related DALYs between countries, which is in line with previous findings about cardiovascular disease risk factors. ${ }^{16}$ For example, although globally only $7.0 \%$ of stroke-related DALYs were associated with alcohol use, in Belarus and Russia the PAF of alcohol use was more than double this proportion $(14.9 \%, 10 \cdot 3-19 \cdot 2$ in Belarus and $14 \cdot 2 \%, 9 \cdot 3-19 \cdot 4$ in Russia; appendix pp 20, 23). Alcohol use has previously been reported to have a significant role in cardiovascular disease burden in Russia. ${ }^{36}$ The betweencountry differences (including differences between highincome and low-income and middle-income countries) in the PAFs of various stroke risk factors might represent differences in sociodemographic factors (eg, population development, migration, or life expectancy), epidemiological factors (eg, variations in the prevalence and management of risk factors), and access to health, social, and community services. Such differences emphasise the need for region-specific approaches to interventions for the reduction of stroke burden.

Our GBD study has several limitations, as described and discussed in detail elsewhere. ${ }^{1}$ For this study, because of the lack of relevant epidemiological data from most countries, we were unable to include some important stroke risk factors (eg, atrial fibrillation and substance abuse) and predisposition to illnesses in the analyses. Inclusion of these and other risk factors might have resulted in an even greater proportion of stroke burden attributable to risk factors and risk factor clusters, because the missing factors are known to have a role in stroke. Also, because of lack of available epidemiological data, we were not able to model different patterns of some risk factors (eg, different dose or types of alcohol drinking; pack-years of smoking; different levels of BMI; elevated blood pressure; or birthweight; genetic factors; socioeconomic status; and public health and medical care interventions) and percentage change in DALYs due to risk factor clusters. Quantification of these other categories of risks or causes is beyond the scope of this study. Additionally, evidence for the selection of TMRELs was uncertain for some risks and based on observational studies that might have had confounders. However, all of our TMREL estimates were discussed and approved by a team of risk epidemiologists and stroke experts. Notably, some risk factors (eg, BMI and diet) have a role in the pathway of stroke rather than being the cause. We did not analyse risk factors for ischaemic and haemorrhagic strokes separately, although another GBD 2013 analysis of 
stroke burden showed great variations in the prevalence of ischaemic and haemorrhagic strokes. ${ }^{3}$ Some risk factors are common for both ischaemic stroke and haemorrhagic stroke, but the contribution of some risk factors differs for different types of stroke. ${ }^{18}$ Although knowledge of risk factors for specific types and causes of stroke is important for the development and implementation of individualised primary prevention strategies, global and regional healthcare policy tends to plan and prioritise stroke prevention based on risk factors for ischaemic and haemorrhagic strokes combined. To address the knowledge gap in GBD, the next step would be to include previous health conditions by age, sex, and stroke subtypes in analyses, in addition to at least some of the broader causes of stroke. Continued regular monitoring of stroke incidence, mortality, prevalence, disability, and modifiable risk factors is important. We also aim to update such crucial evidence for stroke prevention at all levels every 2 years, including geographical regions, type of stroke, and further risk factors.

Our results show that more than $90 \%$ of stroke burden is attributable to modifiable risk factors and that control of behavioural and metabolic risk factors could avert about three-quarters of the global stroke burden. Our data for the global, regional, and country-specific burden of stroke associated with potentially modifiable risk factors and risk factor clusters are important for evidence-based planning, priority setting (including for research), and resource allocation for stroke prevention. Furthermore, these strategies might have similar effects in the prevention of stroke in both males and females, and contribute to the mission of sex equality in health by governments, WHO, and the UN. Our findings about the magnitudes and trends in stroke burden attributable to various modifiable risks and risk clusters suggest that preventive programmes aimed at risk factor modification could lead to major reductions in stroke burden. The age-specific and countryspecific differences in the magnitude of the contributions of various risk factors could inform priorities for stroke prevention from the global level to the national level. For example, reducing exposure to air pollution should be one of the main priorities to reduce stroke burden in lowincome and middle-income countries, whereas reduction of behavioural risks should be the main priority in highincome countries. Also, findings from a systematic review suggest that small increases in fruit and vegetable intake can be achieved effectively in population subgroups. ${ }^{37}$ About half of all strokes could be prevented with effective interventions tackling common lifestyle factors. ${ }^{38}$ Our results have also shown that the contribution of various risk factors to stroke burden varies with time and between countries and continents, thus necessitating regular monitoring of PAFs and country-specific and regionspecific strategies for stroke prevention. In view of the variety of risk factors contributing to stroke burden, preventive strategies should include government policies, health-care providers, non-government organisations, communities, and individuals. Although governments have the power and responsibility to influence environmental, social, medical, and lifestyle factors through legislation and taxation (eg, of tobacco, alcohol, and salt, sugar, or saturated fat content), health service providers have the responsibility to identify risk factors that require medical detection and treatment (eg, elevated blood pressure, atrial fibrillation, dyslipidaemia, or symptomatic carotid artery stenosis) to reduce these risk factors for a substantial proportion of the population. Since many lifestyle habits are set early in life, culturally appropriate education about healthy lifestyles should be incorporated into standardised educational curricula and started early with reinforcement throughout the lifetime. ${ }^{39}$ Multidrug regimens of highly effective and cheap drugs could lead to cost-effective prevention of stroke in lowincome and middle-income regions, potentially halving the risk of death from cardiovascular disease and increasing life expectancy. ${ }^{40}$ Use of far-reaching and affordable technological advances, such as the Stroke Riskometer app—which helps individuals to identify their own risk factors and calculate their future risk of stroke, and provides targeted advice on how to reduce the risk-is another promising approach for stroke prevention worldwide. . $39,42^{2}$ Integrated national health-care systems would need to change to bring patients, health professionals, and carers together to understand the context of the illness and select the best prevention, treatment, and rehabilitation. When cost-effective, individual-level, health-care interventions are complemented by population-wide prevention strategies, a sustainable effect on the global burden of stroke can be achieved. ${ }^{43,44}$ In particular, streamlining of stroke prevention guidelines to take account of the identified behavioural, environmental and occupational, and metabolic risks will help to set out national and international priorities at all levels that can be easily renewed following our global study with updates every 2 years.

Contributors

VLF prepared the first draft. MHF analysed the data and reviewed and edited the first draft and final versions of the manuscript. VLF, GAR MNa, PP, RK, SC, GAM, BN, IS, MNg, KE, KC, CJLM, and MHF reviewed all drafts, finalised the draft, and approved the final version of the manuscript. All other authors provided data, developed models, reviewed results, provided guidance on methodology, reviewed the manuscript, and approved the final version of the manuscript.

\section{Declaration of interests}

VLF, RK, and PP declare that the Stroke Riskometer app is copyrighted by their institution, the Auckland University of Technology, and funds resulting from the sale of the professional version of the app go into further research and education for stroke prevention. $\mathrm{MNg}$ received personal fees from IBM Waton Health. All other authors declare no competing interests.

\section{Acknowledgments}

This research was supported by the Bill \& Melinda Gates Foundation, an American Heart Association Postdoctoral Fellowship to GAR; US National Heart, Lung, and Blood Institute award K08 HL089675-01A1 and a Columbia University Irving Scholarship; and Health Research Council of New Zealand to VLF, RK, and PP. VLF was also partly funded by the Brain Research New Zealand Centre of Research Excellence and 
the "Ageing Well" Programme of the National Science Challenge, Ministry of Business, Innovation and Employment of New Zealand. The views expressed in this Article are those of the authors and do not necessarily represent the views of the National Heart, Lung, and Blood Institute, National Institutes of Health, or the US Department of Health and Human Services.

Global Burden of Diseases, Injuries, and Risk Factors Study 2013 and Stroke Experts Writing Group

Foad Abd-Allah (Department of Neurology, Cairo University, Cairo, Egypt); Semaw Ferede Abera (Food Security Center \& Institute for Biological Chemistry and Nutrition, University of Hohenheim, Stuttgart, Germany); Zanfina Ademi (Institute of Pharmaceutical Medicine, University of Basel, Switzerland and Department of Epidemiology and Preventive Medicine, Monash University, Melbourne, Australia); Rufus Olusola Akinyemi (Neuroscience and Ageing Research Unit, Institute for Advanced Medical Research and Training, College of Medicine, University of Ibadan, Ibadan, Nigeria); Suzanne Barker-Collo (Clinical Training Programme, School of Psychology, The University of Auckland, Auckland, New Zealand); Neeraj Bedi (Faculty of Public Health and Tropical Medicine, Jazan University, Jazan, Saudi Arabia); Derrick A Bennett (Clinical Trial Service Unit and Epidemiological Studies Unit, Nuffield Department of Population Health, University of Oxford, Oxford, UK); Natan M Bornstein (Department of Neurology, Tel-Aviv University, Tel-Aviv, Israel); Michael Brainin (Danube University Krems, Vienna, Austria); Norberto Cabral (Joinville Stroke Registry, University of Joinville, Santa Catarina, Brazil); Ismael Campos-Nonato (Harvard University, Boston, MA, USA); Valeria Caso (Stroke Unit, Department of Cardiovascular and Internal Medicine, Santa Maria della Misericordia Hospital, University of Perugia, Perugia, Italy); Ferrán Catalá-López (Department of Medicine, University of Valencia/ INCLIVA Health Research Institute and CIBERSAM, Valencia, Spain and Ottawa Hospital Research Institute, Ottawa, ON, Canada);

Hanne Christensen (Bispebjerg Hospital and University of Copenhagen, Copenhagen, Denmark); Gabrielle deVeber (Children's Stroke Program, Division of Neurology, Hospital for Sick Children, University of Toronto, Toronto, ON, Canada); Samath D Dharmaratne (Department of Community Medicine, Faculty of Medicine, University of Peradeniya, Sri Lanka and Institute for Health Metrics and Evaluation, Department of Global Health, School of Public Health, University of Washington, Seattle, WA, USA); Eric L Ding (Harvard Chan School of Public Health, Department of Nutrition, Boston, MA, USA, and Microclinic International, Boston, MA, USA); Klara Dokova (Department of Social Medicine and Health Care Organisation, Medical University "Prof Dr Paraskev Stoyanov" Varna, Varna, Bulgaria); Geoffrey A Donnan (The Florey Institute of Neuroscience and Mental Health, and Florey Department of Neuroscience and Mental Health, University of Melbourne, Melbourne, VIC, Australia); Jefferson G Fernandes (Higher School of Health Education and Sciences, Hospital Alemão Oswaldo Cruz, São Paulo, Brazil); Johanna M Geleijnse (Division of Human Nutrition, Wageningen University, Wageningen, Netherlands); Richard F Gillum (Department of Internal Medicine and Department of Community and Family Medicine, Howard University College of Medicine, Washington, DC, USA); Maurice Giroud (Dijon Stroke Registry, Francois Mitterrand University Hospital of Dijon, University Francois Mitterrand University Hospital of Dijon, University of Burgundy, Dijon, France); Rajeev Gupta (Department of Preventive Cardiology and Internal Medicine, Eternal Heart Care Centre and Research Institute, Jaipur, India); Randah R Hamadeh (Arabian Gulf University, Manama, Bahrain); Graeme J Hankey (School of Medicine and Pharmacology, The University of Western Australia, and Department of Neurology, Sir Charles Gairdner Hospital, Nedlands, WA, Australia); Panniyammakal Jeemon (Public Health Foundation of India, New Delhi, India); Jost B Jonas (Department of Ophthalmology, Medical Faculty Mannheim of the Ruprecht-Karls-University of Heidelberg, Mannheim, Germany); Edmond K Kabagambe (Division of Epidemiology, Department of Medicine, Vanderbilt University Medical Center, Nashville, TN, USA); Dhruv S Kazi (Department of Medicine [Cardiology], Department of Epidemiology \& Biostatistics, Global Health Sciences, and Center for Healthcare Value, University of California, San Francisco, CA, USA); Andre Pascal Kengne (South African Medical Research Council and University of Cape Town, Cape Town,
South Africa); Daniel Kim (Department of Health Sciences, Northeastern University, Boston, MA, USA); Yoshihiro Kokubo (Department of Preventive Cardiology, National Cerebral and Cardiovascular Center, Osaka, Japan); Michael A Kravchenko (Research Center of Neurology, Moscow, Russia); Pablo M Lavados (Stroke Unit, Programa Neurología Vascular, Servicio de Neurología, Departamento de Medicina, and Clínica Alemana, Universidad del Desarrollo; Departamento de Ciencias Neurológicas, Facultad de Medicina, Universidad de Chile, Santiago, Chile); Patrice Lindsay (Heart and Stroke Foundation, Ottowa, ON, Canada); Ming Liu (Department of Neurology, West China Hospital, Sichuan University, Chengdu, China); Paulo A Lotufo (Center for Clinical and Epidemiological Research, University of São Paulo, São Paulo, Brazil); Mark T MacKay (Department of Neurology, Royal Children's Hospital and Murdoch Children's Research Institute, Melbourne, VIC, Australia); Man Mohan Mehndiratta (Department of Neurology, Janakpuri Super Speciality Hospital, New Delhi, India); Yohannes Adama Melaku (The University of Adelaide, School of Medicine, Adelaide, SA, Australia); Awoke Misganaw (Institute for Health Metrics and Evaluation, University of Washington, Seattle, WA, USA); Andrew E Moran (Division of General Medicine, Columbia University, New York, NY, USA); Devina Nand (Health Information Unit, Dinem House, Toorak, Suva, Fiji); Grant Nguyen (Institute for Health Metrics and Evaluation, University of Washington, Seattle, WA, USA); Jeyaraj Durai Pandian (Department of Neurology, Christian Medical College, Ludhiana, Punjab, India); Michael A Piradov (Research Center of Neurology, Moscow, Russia); Vasanthan Rajagopalan (School of Allied Health Sciences, Manipal University, Manipal, India); David Rojas-Rueda (ISGlobal, Centre for Research in Environmental Epidemiology, and Universitat Pompeu Fabra, Barcelona, and CIBER Epidemiología y Salud Pública, Madrid, Spain); Ralph L Sacco (Miller School of Medicine, University of Miami, Miami, FL, USA); Ramesh Sahathevan (Universiti Kebangsaan Malaysia Medical Centre [UKMMC], Kuala Lumpur, Malaysia and Malaysia/Ballarat Health Service, Ballarat, VIC, Australia); Kevin N Sheth (Neurocritical Care and Emergency Neurology, Departments of Neurology and Neurosurgery, Yale University School of Medicine, New Haven, CT, USA); Luciano A Sposato (Department of Clinical Neurological Sciences, Western Ontario University, London, ON, Canada);

Konstantinos Stroumpoulis (Anesthesiology Department, Centre Hospitalier Public du Cotentin, Cherbourg, France); David Tanne (Stroke Center, Chaim Sheba Medical Center, Tel Hashomer, Israel); Amanda G Thrift (Department of Medicine, School of Clinical Sciences at Monash Health, Monash University, Melbourne, VIC, Australia); George D Thurston (New York University School of Medicine, Nelson Institute of Environmental Medicine, Tuxedo, NY, USA); David Tirschwell (Harborview Neurology, UW Medicine Comprehensive Stroke Center, Seattle, WA, USA); Thomas Truelsen (Department of Neurology, Copenhagen University Hospital Rigshospitalet, Copenhagen, Denmark); Narayanaswamy Venketasubramanian (Raffles Neuroscience Centre, Raffles Hospital, and Department of Medicine, Yong Loo Lin School of Medicine, National University of Singapore, Singapore); Yury Y Varakin (Research Center of Neurology, Moscow, Russia); Vasiliy Vlassov (National Research University Higher School of Economics, Moscow, Russia); Wenzhi Wang (Department of Neuroepidemiology, Beijing Neurosurgical Institute, Beijing, China); Tissa Wijeratne (Stroke Services and Neuroscience Research Unit, Western Health, Melbourne, VIC, Australia; University of Rajarata, Mihintale, Sri Lanka; University of Melbourne, Melbourne, VIC, Australia; University of Peradeniya, Peradeniya, Sri Lanka); Charles D A Wolfe (Division of Health and Social Care Research, King's College London, and National Institute for Health Research Comprehensive Biomedical Research Centre, Guy's \& St Thomas' NHS Foundation Trust and King's College London, London, UK); Chuanhua Yu (Wuhan University, School of Public Health, Wuhan, Hubei, China); Maigeng Zhou (National Center for Chronic and Non-communicable Disease Control and Prevention of the Chinese Center for Disease Control and Prevention, Beijing, China).

\section{References}

1 GBD 2013 Risk Factors Collaborators. Global, regional, and national comparative risk assessment of 79 behavioural, environmental and occupational, and metabolic risks or clusters of risks in 188 countries, 1990-2013: a systematic analysis for the Global Burden of Disease Study 2013. Lancet 2015; 386: 2287-323. 
2 Murray CJL, Lopez AD. Measuring the global burden of disease. N Engl J Med 2013; 369: 448-57.

3 Feigin VL, Krishnamurthi RV, Parmar P, et al. Update on the global burden of ischemic and hemorrhagic stroke in 1990-2013: the GBD 2013 Study. Neuroepidemiology 2015; 45: 161-76.

4 Roth GA, Johnson CO, Nguyen G, et al. Methods for estimating the global burden of cerebrovascular diseases. Neuroepidemiology 2015; 45: $146-51$.

5 Truelsen T, Krarup LH, Iversen HK, et al. Causes of death data in the Global Burden of Disease estimates for ischemic and hemorrhagic stroke. Neuroepidemiology 2015; 45: 152-60.

6 GBD 2013 Mortality and Causes of Death Collaborators. Global, regional, and national age-sex specific all-cause and cause-specific mortality for 240 causes of death, 1990-2013: a systematic analysis for the Global Burden of Disease Study 2013. Lancet 2015; 385: 117-71.

7 GBD 2013 DALYs and HALE Collaborators. Global, regional, and national disability-adjusted life years (DALYs) for 306 diseases and injuries and healthy life expectancy (HALE) for 188 countries, 1990-2013: quantifying the epidemiological transition. Lancet 2015; 386: 2145-91.

8 SPRINT Research Group. A randomized trial of intensive versus standard blood-pressure control. N Engl J Med 2015; 373: 2103-16.

9 Global Burden of Disease Study 2013 Collaborators. Global, regional, and national incidence, prevalence, and years lived with disability for 301 acute and chronic diseases and injuries in 188 countries, 1990-2013: a systematic analysis for the Global Burden of Disease Study 2013. Lancet 2015; 386: 743-800.

10 Ng M, Fleming T, Robinson M, et al. Global, regional, and national prevalence of overweight and obesity in children and adults during 1980-2013: a systematic analysis for the Global Burden of Disease Study 2013. Lancet 2014; 384: 766-81.

11 Fowkes FGR, Rudan D, Rudan I, et al. Comparison of global estimates of prevalence and risk factors for peripheral artery disease in 2000 and 2010: a systematic review and analysis. Lancet 2013; 382: 1329-40.

12 Feigin VL, Forouzanfar MH, Krishnamurthi R, et al. Global and regional burden of stroke during 1990-2010: findings from the Global Burden of Disease Study 2010. Lancet 2014; 383: 245-54.

13 WHO. Global health risk: mortality and burden of disease attributable to selected major risks. Geneva: World Health Organization, 2009.

14 Malik VS, Willett WC, Hu FB. Global obesity: trends, risk factors and policy implications. Nat Rev Endocrinol 2013; 9: 13-27.

15 Cesare MD, Bennett JE, Best N, Ezzati M, Stevens GA, Danaei G. The contributions of risk factor trends to cardiometabolic mortality decline in 26 industrialized countries. Int J Epidemiol 2013; 42: 838-48.

16 Yusuf S, Rangarajan S, Teo K, et al. Cardiovascular risk and events in 17 low-, middle-, and high-income countries. N Engl J Med 2014; 371: 818-27.

17 WHO. The world health report 2002-reducing risks, promoting healthy life. Geneva: World Health Organisation, 2002. http://www. who.int/whr/2002 (accessed Jan 16, 2016).

18 O'Donnell MJ, Xavier D, Liu L, et al. Risk factors for ischaemic and intracerebral haemorrhagic stroke in 22 countries (the INTERSTROKE study): a case-control study. Lancet 2010; 376: 112-23.

$19 \mathrm{Hu} \mathrm{G}$, Tuomilehto J, Silventoinen K, Sarti C, Männistö S, Jousilahti P. Body mass index, waist circumference, and waist-hip ratio on the risk of total and type-specific stroke. Arch Intern Med 2007; 167: 1420-27.

20 Kontogianni MD, Panagiotakos DB. Dietary patterns and stroke: a systematic review and re-meta-analysis. Maturitas 2014; 79: 41-47.

21 He FJ, Li J, MacGregor GA. Effect of longer term modest salt reduction on blood pressure: Cochrane systematic review and meta-analysis of randomised trials. BMJ 2013; 346: f1325

22 Lock K, Pomerleau J, Causer L, Altmann DR, McKee M. The global burden of disease attributable to low consumption of fruit and vegetables: implications for the global strategy on diet. Bull World Health Organ 2005; 83: 100-08.
23 Pierce JP, Messer K, White MM, Cowling DW, Thomas DP. Prevalence of heavy smoking in California and the United States, 1965-2007. JAMA 2011; 305: 1106-12.

24 Gallus S, Lugo A, La Vecchia C, et al. Pricing policies and control of tobacco in Europe (PPACTE) project: cross-national comparison of smoking prevalence in 18 European countries. Eur J Cancer Prev 2014; 23: 177-85.

25 Liu Y, Gao J, Shou J, et al. The prevalence of cigarette smoking among rural-to-urban migrants in China: a systematic review and meta-analysis. Subst Use Misuse 2016: 51: 1-10.

26 Chen Z, Peto R, Zhou M, et al. Contrasting male and female trends in tobacco-attributed mortality in China: evidence from successive nationwide prospective cohort studies. Lancet 2015; 386: 1447-56.

27 Woodward M, Martiniuk A, Ying Lee CM, et al. Elevated total cholesterol: Its prevalence and population attributable fraction for mortality from coronary heart disease and ischaemic stroke in the Asia-Pacific region. Eur J Cardiovasc Prev Rehabil 2008; 15: 397-401.

28 Barker-Collo S, Bennett DA, Krishnamurthi RV, et al. Sex differences in stroke incidence, prevalence, mortality and disability-adjusted life years: results from the Global Burden of Disease Study 2013. Neuroepidemiology 2015; 45: 203-14.

29 Shah ASV, Lee KK, McAllister DA, et al. Short term exposure to air pollution and stroke: systematic review and meta-analysis. BMJ 2015; 350: h1295.

30 Brauer M, Freedman G, Frostad J, et al. Ambient air pollution exposure estimation for the Global Burden of Disease 2013. Environ Sci Technol 2016; 50: 79-88.

31 Ostro B, Hu J, Goldberg D, et al. Associations of mortality with long-term exposures to fine and ultrafine particles, species and sources: results from the California Teachers Study Cohort. Environ Health Perspect 2015; 123: 549-56.

32 Navas-Acien A, Guallar E, Silbergeld EK, Rothenberg SJ. Lead exposure and cardiovascular disease-a systematic review. Environ Health Perspect 2007; 115: 472-82.

33 Kengne AP, Anderson CS. The neglected burden of stroke in Sub-Saharan Africa. Int J Stroke 2006; 1: 180-90.

34 Moosa MR, Van Der Walt I, Naicker S, Meyers AM. Important causes of chronic kidney disease in South Africa. S Afr Med J 2015; 105: 2681

35 Ding EL, Malik VS. Convergence of obesity and high glycemic diet on compounding diabetes and cardiovascular risks in modernizing China: an emerging public health dilemma. Global Health 2008; 4 : 4

36 Zaridze D, Brennan P, Boreham J, et al. Alcohol and cause-specific mortality in Russia: a retrospective case-control study of 48557 adult deaths. Lancet 2009; 373: 2201-14

37 Pomerleau J, Lock K, McKee M. Interventions designed to increase adult fruit and vegetable intake can be effective: a systematic review of the literature. J Nutr 2005; 135: 2486-95.

38 Tikk K, Sookthai D, Monni S, et al. Primary preventive potential for stroke by avoidance of major lifestyle risk factors: the European Prospective Investigation Into Cancer and Nutrition-Heidelberg cohort. Stroke 2014; 45: 2041-46.

39 Norrving B, Davis SM, Feigin VL, Mensah GA, Sacco RL, Varghese C. Stroke prevention worldwide-what could make it work. Neuroepidemiology 2015; 45: 215-20.

40 Gaziano TA, Opie LH, Weinstein MC. Cardiovascular disease prevention with a multidrug regimen in the developing world: a cost-effectiveness analysis. Lancet 2006; 368: 679-86.

41 Feigin VL, Krishnamurthi R, Bhattacharjee R, et al. New strategy to reduce the global burden of stroke. Stroke 2015; 46: 1740-47.

42 Morgan J. The power of the App: can mobile-technology save lives? Lancet Neurol 2015; 15: 668.

43 WHO. Global status report on noncommunicable diseases 2010. Description of the global burden of NCDs, their risk factors and determinants. Geneva: World Health Organization, 2011.

44 Collins FS, Varmus H. A new initiative on precision medicine. N Engl J Med 2015; 372: 793-95. 